\title{
Sprachwissenschaft und öffentliche Sprachdiskus- sion - Wurzeln ihres problematischen Verhältnisses
}

\section{Ein problematisches Verhältnis}

Einen Tag nach Abschluß der letztjährigen Jahrestagung des Instituts für deutsche Sprache über die Tendenzen und Perspektiven der deutschen Gegenwartssprache erschien auch in meiner Tageszeitung ein dpaBericht über die Vorträge und Diskussionen. Er trug die Überschrift „Wissenschaftler widersprechen These vom Verfall der deutschen Sprache" (1989). Der Inhalt entspricht der Schlagzeile. Ins Zentrum des Interesses wird die Frage nach dem Zustand der deutschen Sprache gerückt, und es kommen die "Professoren Grosse, Wimmer und Stickel" mit ihrer Auffassung zu Wort, die deutsche Sprache lebe und werde reicher, sei auch insgesamt gut in Schuß und die These vom Sprachverfall vermutlich in erster Linie ein Generationsproblem. Der einzige Vortrag, über den inhaltlich berichtet wird, ist der von Sitta über das gestiegene Schreibvermögen von Gymnasiasten und Studenten (vgl. Sitta 1990). Am folgenden Tag erscheint eine Glosse zu dem Agenturbericht, betitelt "Alterserscheinungen" in Anspielung an die These vom Sprachverfall als Generationsproblem. Der Autor -thes" (Matthes 1989) kann kaum glauben, was dpa getickert hat und schließt die Möglichkeit nicht aus, daß der journalistische Kollege die zitierten Wissenschaftler vielleicht hier und da mißverstanden hat. Auf das Alter freilich will er nichts kommen lassen, es schütze zwar vor Torheit nicht, verschaffe aber bessere Vergleichsmöglichkeiten. Diese nutzend, kommt er zum Ergebnis: „Ob man es Sprachverfall nennen soll, wenn heutige Abiturienten und Akademiker die Rechtschreibsicherheit früherer Volksschulabsolventen nicht erreichen, mag offenbleiben. Sicher aber gehört es nicht zu dem, was heute besser ist als früher".

Dieses kleine Zeugnis öffentlicher Berichterstattung über eine sprachwissenschaftliche Tagung mag genügen, um andeutend zu begründen, warum ich das Verhältnis zwischen Sprachwissenschaft und öffentlicher Sprachdiskussion im Titel "problematisch" genannt habe. Der Vorgang weist typische Züge auf. Das Nachdenken über Sprache findet in unterschiedlichen Räumen und auf unterschiedlichen Foren statt; von einigen Grenzgängern abgesehen sind es unterschiedliche Gruppen von Personen, die sich auf den recht streng getrennten Foren bewegen; die Verständigung zwischen ihnen ist mühsam, wenn nicht unmöglich; die Auseinandersetzung zwischen ihnen stark polemisch geprägt, und das letzte Wort haben in aller Regel nicht die Sprachwissenschaftler. 
Der historische Rückblick hat, insofern als nach den Wurzeln eines gegenwärtig problematischen Verhältnisses gefragt wird, die Funktion, aus der historischen Analyse Hinweise darauf zu gewinnen, wann und aus welchen Gründen sich die Trennung einer wissenschaftlichen und einer öffentlichen Sprachdiskussion ausgebildet hat, inwieweit sie notwendig war und ist und inwieweit eventuell aufhebbar.

\section{Sprachwissenschaft und öffentliche Sprachdiskussion in der ersten Hälfte des 19. Jahrhunderts}

Von einem Verhältnis zwischen $A$ und $B$ kann man erst dann sprechen, wenn die Elemente, die in das Verhältnis eingehen, eine gewisse Eigenständigkeit gewonnen haben. Diese Voraussetzung entwickelt sich im vorliegenden Falle in den ersten Jahrzehnten des 19. Jahrhunderts mit der Entstehung und Etablierung der Sprachwissenschaft in institutionalisierter Form als akademischer Disziplin in Gestalt der historischen und vergleichenden Sprachforschung (vgl. Bahner/Neumann 1985). Auf ein Datum will ich mich nicht, brauche ich mich auch nicht festzulegen; sicher ist aber, daß schon um 1820 eine ausgesprochene Spannung zwischen der sich ausbildenden historischen Sprachwissenschaft und dem heterogen strukturierten Bereich der Sprachdiskussion im Umkreis der Schule und der Sprachdidaktik, der Sprachgesellschaften und dem weiten Feld der Publizistik besteht. Und zwar ist diese Spannung nicht voll zu erklären mit dem in allen Wissensgebieten zu beobachtenden Faktum, daß die Professionalisierung und Institutionalisierung wissenschaftlicher Disziplinen die öffentliche Rezeption und Verwertung ihrer Ergebnisse erschwert oder gar verhindert und die für die jeweiligen Gegenstände nicht speziell Ausgebildeten, aber gleichwohl Interessierten, in die Rolle der Autodidakten oder der mit einiger Geringschätzung betrachteten Laien abdrängt.

Die Geschichtsschreibung der Sprachwissenschaft hat lange im eingeengten Blick auf die historische Sprachforschung eine starke Um- und Neuorientierung vom 18. zum 19. Jahrhundert wahrgenommen und das, was sich diesem Bild nicht fügte, als Auslaufendes, an die Peripherie Gedrängtes, wissenschaftlich Überholtes eingeschätzt. Was u.a. als auslaufend wahrgenommen wurde, zeigt das folgende Zitat:

Als communis opinio deutscher Sprachgeschichtsforschung kann [...] angesehen werden, daß im Spektrum der vielfältigen Sprachbewegungen des 18. Jahrhunderts die Herausbildung einer weithin anerkannten, in Literatur und Wissenschaft brauchbaren Schriftsprache das herausragende Ereignis ist [Blackall 1966]. Dem trägt auch die sprachwissenschaftliche Praxis dieser Zeit Rechnung: Am Ende des Jahrhunderts kommt es (aus heutiger Sicht folgerichtig) zu einem gewissen Abschluß 
der lange währenden sprachkritischen Diskussionen und zur Festschreibung und Dokumentation der neuen Schriftsprache als Standardsprache durch Lexikographie und Grammatikographie anerkannter Autoritäten [Cherubim 1983, S. 402] .

Wenn man bei „sprachwissenschaftlicher Praxis” z.B. an Jacob Grimm denkt, so hat die referierte Meinung viel für sich. Nimmt man jedoch insgesamt ins Blickfeld, was die Zeitgenossen in der ersten Jahrhunderthälfte bewegt hat, so bekommt man ein ganz anderes Bild: das Bild einer breit angelegten Diskussion über die deutsche Gegenwartssprache und die Notwendigkeit ihrer weiteren Ausbildung, Vervollkommnung und Verbreitung in deutlichem Anschluß an die aufklärerischen Traditionen des 17. und 18. Jahrhunderts, eher Kontinuität also als Umbruch. Überspitzt könnte man sogar sagen, daß es die sich etablierende historische und vergleichende Sprachwissenschaft war, die sich aus dem gesamtgesellschaftlichen, gegenwartsbezogenen Diskussionszusammenhang, der die Reflexion über die deutsche Sprache im 18. Jahrhundert charakterisierte, aussonderte.

Aber welche Probleme bestanden denn noch? War denn nicht in der Tat inzwischen eine "weithin anerkannte, in Literatur und Wissenschaft brauchbare Schriftsprache" entstanden und auch in Lexikographie und Grammatikographie normativ festgeschrieben worden? - Nimmt man den Sammelband "Reichthum und Armut deutscher Sprache" (Dieckmann 1989) zur Hand, so findet man in der ersten Hälfte des Jahrhunderts kaum jemanden, der sich im wohlgefälligen Blick auf das von den Klassikern Erreichte im Lehnstuhl zurücklehnte. Die Klage über den bedauernswerten Zustand der deutschen Sprache herrscht vor, und das Ziel ist, hier mehr national, dort mehr liberal motiviert, nicht Konservierung, sondern zukunftsgerichtet weitere Entwicklung, Ausbildung und Vervollkommnung. Worin bestehen die Mängel? Sie lassen sich, aus einiger Entfernung von den einzelnen Autoren (z.B. A. Müller, Jahn, Arndt, Körner, F. Schlegel, Jochmann, Wienbarg, Mundt, Auerbach) betrachtet, wie folgt zusammenfassen:

(1) Wir haben inzwischen eine nationale Sprache in der schönen Literatur und in der Philosophie und den Wissenschaften, aber nur dort. Insbesondere fehlt uns eine öffentliche Sprache für die notwendige Diskussion der großen gesellschaftlichen und politischen Fragen der Zeit, auch eine Sprache des öffentlichen geselligen Verkehrs. - Das erste Problem ist also die eingeschränkte funktionale Geltung der bestehenden Standardsprache. Im allgemeinen herrscht ein klares Bewußtsein davon, daß sich in einem bestimmten Kommunikationsbereich eine gemeinsame Sprache nur in der kommunikativen Praxis ausbilden könne, daß man also nicht 
den Sprachstil eines bestimmten Bereichs, etwa der Literatursprache, auf einen anderen übertragen kann. Der Mangel auf der sprachlichen Ebene wird als eng verknüpft mit einem gesellschaftlichen Übelstand wahrgenommen.

(2) Die geschriebene Standardsprache in ihrer funktional begrenzten Geltung ist Besitz einer schmalen Schicht der Bevölkerung, nicht aber die Sprache des Volkes, nicht einmal allgemein verwendetes Medium des Verkehrs in den oberen Gesellschaftsschichten. - Das zweite Problem ist also die extrem eingeschränkte soziale Geltung der geschriebenen Standardsprache.

(3) Wir haben, funktional und sozial stark eingeschränkt, eine geschriebene, aber keine gesprochene Sprache.

(4) Die Sprache, die wir haben, ist eine vor allem von den Wissenschaften geprägte Büchersprache, deren Charakter die notwendige Erweiterung des Gebrauchs behindert.

Die Mängelliste zeigt, daß im Vergleich mit der zweiten Hälfte des 18. Jahrhunderts inzwischen nicht mehr Normierung und Kodifizierung als Hauptproblem gilt, sondern Erweiterung des standardsprachlichen Gebrauchs vordringlich geworden ist, wiewohl auch dieses sprachpolitische Programm sich in die Tradition aufklärerisch-volkspädagogischer Bestrebungen stellen läßt. Das Normproblem taucht in diesem Zusammenhang in unterschiedlicher Weise auf, je nachdem, wie die wünschenswerte funktionale und soziale Erweiterung nach Auffassung der jeweiligen Autoren bewerkstelligt werden soll. Wer wie Jahn und manche Nachfolger bis hin zu Auerbach in der Jahrhundertmitte die Büchersprache durch Rückgriff auf die Dialekte und die Prinzipien der gesprochenen Sprache oder auf historische Sprachstufen verlebendigen will, muß es sich angelegen sein lassen, die Gottschedschen und Adelungschen Festschreibungen, z.B. in der Frage der Dialektismen und der Archaismen, zu lockern oder rückgängig zu machen. Andererseits ist nicht zu übersehen, daß für die Aufgabe, die Geltung der Standardsprache über das Bildungsbürgertum hinaus zu erweitern, dem Sprachunterricht eine wesentliche Rolle zufiel, und daß die schulische Erlernung einer nur rudimentär oder gar nicht im sprachlichen Verkehr erworbenen Sprache Normen für den richtigen und guten Gebrauch voraussetzt. Außerdem übernahm der Deutschunterricht an Gymnasien im gleichen Zeitraum zunehmend Aufgaben des Grammatikunterrichts und der Rhetorik, die vormals mit dem Lateinunterricht verknüpft waren (vgl. Abels 1988), was die normative Tendenz unterstützte. Da die sich etablierende Germanistik für solche Aufgaben keine Hilfe anbot, stützten sich die zeitgenössischen Grammati- 
ker und Rhetoriker weiterhin auf die älteren Traditionen der normativen und der philosophischen Grammatik, ja die Prinzipien der philosophischen Sprachforschung gewannen in den ersten Jahrzehnten des 19. Jahrhunderts sogar noch einmal verstärkt Geltung (vgl. Naumann 1986). Erst nach der Mitte des Jahrhunderts gibt es einen deutlichen EinfluB der historisch-vergleichenden Sprachwissenschaft auf die Schulgrammatik und den Sprachunterricht.

Man kann nun zwar von den Grammatikern, wie in anderer Weise von den Philosophen, Dichtern, Publizisten, die sich an der Diskussion über die gegenwärtige deutsche Sprache und die Möglichkeiten zu ihrer Veränderung beteiligten, sagen, sie befänden sich wissenschaftlich nicht auf der Höhe der Zeit; doch ist das Argument schwach, wenn man als Maßstab für die Wissenschaftlichkeit die historische Sprachwissenschaft nimmt. Relativ zu den Problemen, die in der öffentlichen Sprachdiskussion thematisiert werden, und auch denen, für die die grammatischen, stilistischen und rhetorischen Lehrwerke geschrieben werden, stellen die Prinzipien und Methoden der historischen Sprachwissenschaft keine geeignete Grundlage dar.

Akademische Sprachwissenschaft und öffentliche Sprachdiskussion sind in der ersten Jahrhunderthälfte sicherlich nicht unverbundene, jedoch schon deutlich getrennte Bereiche der Reflexion über Sprache, und beide sind es aus eigenem Recht.

\section{Aspekte öffentlicher Sprachdiskussion seit ca. $\mathbf{1 8 5 0}$}

\subsection{Sprachkritik im Dienst sozialer Abgrenzung}

Dies, das eigene Recht, auch der Sprachkritik der zweiten Jahrhunderthälfte zuzubilligen, fällt schwerer, $z u$ stark ist sie von sekundären Motiven, besonders von dem Bemühen um soziale Distinktion überlagert. Die Sprachsituation hatte sich schon um die Jahrhundertmitte verändert. Zwar kann man nicht sagen, daß die gesellschaftlichen Aufgaben, die die liberalen und nationalen Sprachkritiker der ersten Jahrhunderthälfte mit ihrem Programm der funktionalen und sozialen Erweiterung zu lösen versucht hatten, inzwischen gelöst waren; doch hatte sich die Standardsprache in vielen Kommunikationsbereichen zunehmend durchgesetzt, und es hatte sich die Schicht derer, die die Standardsprache aktiv und passiv beherrschten, erweitert. Die Sprachkritiker reagierten aber nun gerade auf diese veränderte Situation mit einer entschiedenen Kritik und mit einer zum Teil heftigen Polemik gegen die Träger negativ bewerteter Sprachformen. Sprachkritik verband sich in problematischer Weise mit dem Willen zu sozialer Abgrenzung, und in dieser Funktion rückte die 
Frage nach dem richtigen und falschen Sprachgebrauch, die in der ersten Jahrhunderthälfte nicht das dominante Thema gewesen war, erneut ins Zentrum der öffentlichen Sprachdiskussion.

Wer grenzt sich gegen wen $a b$ ? In der Literatur zur Sprachpflege und Sprachkritik im 19. Jahrhundert herrscht die Meinung vor, das Bürgertum grenze sich mit sprachpflegerischen Mitteln von den unteren Schichten $a b$, die "Standardsprachenideologie " unterstütze die "Abgrenzung von Bürgertum und Arbeiterschaft" (Cherubim/Mattheier 1989, S. 7). Das ist wohl nicht ganz richtig. Die Angst des Bürgers vor Pöbelherrschaft und Anarchie, deutlich Ausdruck findend schon zu den Zeiten revolutionärer Bewegungen nach 1789 und um 1830 und 1848, und die gesellschaftliche und politische Frontstellung gegen die sich organisierende Arbeiterbewegung in der zweiten Jahrhunderthälfte kann zwar nicht bezweifelt werden, genausowenig wie entsprechende Tendenzen in der Bildungs- und Schulpolitik im gesamten Zeitraum; das in der Sprachkritik sich ausdrückende Abgrenzungsbedürfnis richtet sich jedoch nicht gegen das städtische und ländliche Proletariat. Angemessener ist es, im Sinne Wehlers (1987, bes. Kap. III, S. 4) von einer " ${ }^{\text {Sozialdefensiven }}$ Maßnahme" im "innerbürgerlichen Konkurrenzkampf" zu sprechen. Die Großfamilie des eigentlichen Bildungsbürgertums, die akademisch geschulte, meist staatlich angestellte Intelligenz in Bürokratie, Kirche, Universitäten und Gymnasien, verteidigte ihre Vormachtstellung gegen andere Fraktionen des Bürgertums, die zunehmend an gesellschaftlicher Geltung gewannen: das Handels- und Besitzbürgertum, die technische Intelligenz, die Angehörigen der sich ausbildenden freien Berufe.

Diese Stoßrichtung ist einfach zu erklären, wenn man bedenkt, worauf die soziale Distinktion des Bildungsbürgertums beruht hatte: auf Bildung eben, die zudem im neuhumanistischen Verständnis in erster Linie philosophisch-historisch-literarische Bildung war und ganz zentral die Beherrschung der Standardsprache voraussetzte. Das Merkmal der Beherrschung der Standardsprache verlor jedoch mit der faktischen Erweiterung ihres Gebrauchs zunehmend seinen unterscheidenden Wert. Die bildungsbürgerliche Exklusivität wurde aber im gesamten Jahrbundert nicht von den "niederen", sondern von den nachrückenden bürgerlichen Schichten bestritten. So darf man sich von den nicht seitenen Ausfallen Schopenhauers gegen den "Pöbel" nicht in die Irre führen lassen; es ist durchweg bürgerlicher Pöbel, der in den 30er und 40er Jahren die Sprache verhunzt hat. Die ${ }_{n}$ ganz jämmerlichen, winzigen, schwindsüchtigen, mißgeschaffenen Menschen" (Schopenhauer 1985, Bd. 1, S. 352) mit „flachen Schädeln, aus denen trübe Schweinsaugen spähen" (Bd. 4, I, S. 241), mit „kahlen Köpfen, langen Bärten, Brillen statt der Augen, als 
Surrogat der Gedanken ein Cigarro im thierischen Maul" (Dieckmann 1989, S. 241), „die langbärtigen $Z$ werge, die überall euch $z$ wischen die Beine laufen, und deren Eltern schon bloB aus Gnaden der Kuhpocken am Leben geblieben sind" (ebenda, S. 247), sind allesamt bürgerlich. Schopenhauer findet oder vermutet solche Exemplare unter den philosophischen Kollegen, unter den Wissenschaftlern, unter den Angehörigen der freien Berufe (Ärzten, Advokaten, Richtern und Berufsjournalisten) und in der technischen Intelligenz, die er zum Volk rechnet: „Man soll nicht vergessen, daß jeder Mensch, der kein Latein versteht, zum Volke gehört, auch wenn er ein großer Virtuose auf der Elektrisirmaschine ist und das Radikal der Fluß[spath]säure im Tiegel vorzeigen könnte" (Schopenhauer 1985, Bd. 4, II, S. 79). Das Latein ist auch nötig, um richtig Deutsch sprechen und schreiben zu können, nämlich z.B. nicht nitaliehnisch" mit langem geschlossenen $e$, geschrieben $e,{ }_{n}$ wie ein Dreckfeger", sondern, da abgeleitet von lat. Italia, mit offenem $e$, geschrieben $\vec{a}$ (Bd. 4, II, S. 37).

Vierzig Jahre später, bei Wustmann (1912, S. 21f. u.ö.), taucht das "niedrige Volk", das „unwissende", „faule", auch „plebejische", schon eher als Träger der bekämpften Sprachdummheiten auf. Den Beispielen, bei denen er sich gegen die der "feinern Umgangssprache" abträglichen Sprachformen der Ladendiener und Ladenmädchen und der Gassensprache wendet, stehen jedoch etwa gleich viele gegenüber, bei denen er den gesunden sprachlichen Sinn und die Lebendigkeit der Sprache des Volkes, besonders wenn es sich auf obersächsischen Gassen tummelt, gegen das "Papierdeutsch" ins Feld führt. Der Hauptgegner ist immer noch der halbgebildete Bürger, der den Amtsstil, den Zeitungsstil und den Geschäftsstil verantwortet, nicht zu vergessen freilich die "Herren Pädagogen", die Volksschullehrer. - Die Einschätzung der Sprache der Unterschicht ist bei allen, die sich - wie Hildebrand (1867), Wustmann (1912), Schroeder (1906), Engel (1922) u.a. - zum Anwalt einer "Sprache des warmen, tätigen Lebens" (Schroeder) gegen den „Papierstil" und den „schablonenhaften" Sprachgebrauch (Engel) machen, ambivalent. Einerseits wollen sie die feinere Umgangssprache von plebejischen Sprachformen freihalten, andererseits brauchen sie aber die Sprache des Volkes, um die Erstarrungen der zeitgenössischen Schriftsprache aufzulösen. Der Ambivalenz wird man vielleicht mit der Charakterisierung gerecht: Sie lieben nicht das Volk, aber wenigstens den Volksmund.

Eines der klarsten Programme für Sprachpflege im Dienste sozialer Abgrenzung stammt nicht aus dem 19. Jahrhundert, sondern wurde vor nunmehr 23 bzw. 24 Jahren den Teilnehmern einer Jahrestagung dieses Instituts von Süskind präsentiert. Er spricht von der Qual, 
die es einem empfindlichen Menschen auch schon bereiten kann, wenn er in einem öffentlichen Lokal die Katarakte eines frechen, brülligen, wie auf ständige Zoten reagierenden Gelächters vernehmen muß, aus denen sich so eine mittelbürgerliche Sonntagsabendkonversation vorzüglich zusammenzusetzen scheint. Ich spreche von Qual - es ist auch Verzweiflung dabei, was alles sich an Rohheit und frechem Neureichenthum in unserer Gesellschaft laut- und breitmacht. Und solcher Verzweillung, mutatis mutandis, müßte auch einer Sprachplege heute einiges innewohnen - Verzweiflung darüber, daß Ordnungen gestört sind. (Süskind 1968, S. 192).

Auf Abhilfe sinnend, läßt er seinen Blick nach England schweifen, wo die kodifizierte Norm des "King's English", so meint er, "bis heute mit wahrhaft akademischer Gewalt" entscheidet, „wo ein Mensch, wenn er den Mund auftut, hingerechnet wird" (ebda., S. 194). Ähnliches erhofft er sich von der Sprachpflege auch in Deutschland: „eine ins Gesellschaftliche, ganz allgemein ins Politische ausstrahlende Wirkung" (S. 194). Welche denn wohl? Offenbar Normbestimmungen, die die Sortierung der Sprecher in die Gruppe derer ermöglicht, die das gutbürgerliche Lokal am Markt am Sonntagabend rechtmäßig besuchen, und in die Gruppe derer, die sich dort zu Unrecht breitmachen.

Nichts Schlimmeres könnte einer so motivierten Sprachkritik passieren, als wenn die Mischpoke sich tatsächlich den gewünschten Gebrauchsweisen der Sprache anbequemen würde! Das steht indes nicht zu befürchten. Die sprachlichen Phänomene, die inzwischen zur "Hinrechnung" vorzugsweise verwendet werden, sind so geartet, daf man ihnen nicht ohne sprachliche Verrenkungen gerecht werden kann, so daß sie auch in $\mathrm{Zu}$ kunft unbegrenzt zur Verfügung stehen werden. Sie sind das Endprodukt einer zunehmenden Verengung des sprachkritischen Horizontes als Folge einer immer höher angelegten Meßlatte.

Am Anfang des 19. Jahrhunderts konnte die Frage, wer als sprachlich gebildet gelten sollte, im wesentlichen mit dem Kriterium der standardsprachlichen Lese- und Schreibfähigkeit entschieden werden. Mit der zunehmenden Ausbreitung dieser Fähigkeit verlagerte sich die Unterscheidung zum einen auf die zusätzliche Beherrschung einer zumindest standardnahen Umgangssprache. In Hinblick auf die geschriebene Sprache bekam die Forderung nach Freiheit von Dialektismen, Regionalismen und Umgangssprachlichem verstärkt Gewicht, besonders aber die Verpflichtung auf die spezifischen Stiltraditionen der klassischen deutschen Literatur, die in der Sprachkritik der zweiten Hälfte des 19. Jahrhunderts bekanntermaßen eine große Rolle spielt.

Zur Gegenwart hin hat sich das Spektrum der Gegenstände, die Anlaß sprachpflegerischer Bemühungen werden, aber noch weiter verengt - 
auch verglichen z.B. mit Wustmann. Ich habe mir die ca. zwanzig Taschenbücher der letzten zwanzig Jahre, die meine heimische Seminarbibliothek enthält und deren Verfasser ihr Augenmerk ausdrücklich auf das richtige und gute Deutsch richten, die heutigen Wustmänner also, etwas genauer angesehen. Solch ein Buch enthält typischerweise 30, 50, höchstens 70 Artikel zu einzelnen grammatischen oder lexikalischen Problemen. Unter ihnen befinden sich, mehr oder weniger vollständig, die 2.T. 100 - 200 Jahre alten sprachkritischen Ladenhüter: derselbe und der gleiche, scheinbar und anscheinend, lehren und lernen, auf und offen, hoch und hinauf, hin und her, als und wie, brauchen ohne zu, Genetiv oder Dativ nach trotz oder wegen, trotzdem als subordinierende Konjunktion, Umschreibung des Konjunktivs mit würde, Verwechslung der Vergangenheitstempora und eine Handvoll andere. Obligatorisch ist ferner eine Reihe von Artikeln zu Sprachformen, deren richtige Verwendung im Deutschen, folgt man der Argumentation der Sprachkritiker, beim Sprachbenutzer die Kenntnis fremder Sprachen voraussetzt. Auch unter den wirklichen oder vermeintlichen Pleonasmen sind besonders die Fremdwörter beliebt, der Werbeprospekt auf der Glasvitrine, das vorprogrammierte Scheitern des Testversuchs und das maschinengeschriebene Manuskript, deren pleonastischer Charakter sich nur dem erschließt, der die fremdsprachliche Verwendung des Wortes kennt und dem Argument zu folgen bereit ist, daß sich der Gebrauch eines Fremdwortes im Deutschen formal und inhaltlich am Gebrauch in der Ursprungssprache zu orientieren habe. Eine gewisse Variation weist eine dritte Abteilung auf, bestehend aus Modewörtern und Neologismen, und eine Restgruppe, die ich als "Sonstiges" auf sich beruhen lasse. Die Glossen, die in solchen Büchern gesammelt werden und meistens aus den Sprachecken von Zeitungen stammen, betreffen inzwischen überwiegend oder sogar ausschließlich Sprachformen, deren "richtige" Verwendung im faktischen Sprachgebrauch, auch dem der Standardsprecher, nicht sicher verankert ist und deshalb auch nicht im sprachlichen Verkehr, sondern nur in formellen Lernsituationen oder durch Lektüre sprachkritischer Bücher erworben werden kann. Und das genau macht die Voraussage sicher, daß die Ladenhüter den Sprachkritikern auch in Zukunft erhalten bleiben werden, und mit ihnen die Dummen, über die sich auch die Leser und Leserinnen erheben können. Fast alle Zeitungen versichern ja glaubhaft, mit welcher Zustimmung die Leser die Arbeit der Glossisten leserbriefschreibend begleiten. Sie freilich können die Sprüche vom hin und her und vom brauchen ohne $z u$ längst auswendig. Gemeint sind immer die anderen. 


\subsection{Kriterien zur Bestimmung der Sprachrichtigkeit}

Der erneute Hinweis auf die Rolle der Sprachkritik in der Verteidigung bildungsbürgerlichen Sprachkapitals zeigt, daß die Frage nach dem richtigen und guten Deutsch bis heute von einem sachfremden Gesichtspunkt überlagert ist. Er erlaubt aber noch kein Urteil über Sinn und Nutzen der Frage nach dem richtigen Deutsch und über die Kriterien, auf Grund deren Richtigkeit festgestellt und gegebenenfalls normativ gefordert wird. Die Kriterien sind, auch unabhängig davon, daß sie zu sozialdistinktiven Zwecken verwendet werden, zwischen Sprachwissenschaft und Sprachkritik strittig. Sie sollen Gegenstand des letzten Vortragsteils sein.

Die erste Beobachtung, die man bei einer systematischen Lektüre von Sprachglossen macht, ist, daß eine einigermaßen konsistente Beurteilungsgrundlage offenbar nicht vorhanden ist. Von Fall zu Fall wählt der Sprachkritiker andere Kriterien oder kombiniert verschiedenartige aus einem Reservoir, das vor allem die folgenden Möglichkeiten enthält: (1) die Berufung auf eine Autorität (z.B. den Duden oder das, was man in der Schule gelernt hat), (2) die Berufung auf den gegenwärtigen Sprachgebrauch, (3) die Berufung auf den früheren Sprachgebrauch, (4) die Berufung auf bestimmte auszuzeichnende Sprechergruppen (z.B. die guten Schriftsteller der Gegenwart oder der Vergangenheit), (5) die Berufung auf eine erhöhte Funktionalität $(z . B$. die Möglichkeit semantischer Differenzierung) und (6) die Berufung auf die Struktur der Wirklichkeit bzw. das diese Wirklichkeit "sachgemäf”, "logisch", „richtig” erfassende Denken. Häufig werden die Argumente auch gehäuft, ohne daß klar ist, welches Kriterium die Beweislast trägt und welche zusätzliche Unterstützung liefern sollen. Für den linguistischen Betrachter irritierend ist ferner die geringe Verallgemeinerbarkeit der vorgebrachten Argumente bzw. die Unsicherheit darüber, ob der Kritiker selbst sie für verallgemeinerbar hält. Wenn propagandieren falsch ist, weil der lateinische Stamm propag- lautet, ist dann auch Propagandist falsch oder nicht? Wenn aber nicht, warum soll für die eine Ableitung etwas anderes gelten als für die andere? Man wird häufig den Verdacht nicht los, daß die Autoren schon $\mathrm{zu}$ wissen glauben, was richtig ist, und sekundär sich etwas Passendes aus dem Topf möglicher Argumente aussuchen.

In der bisherigen sprachwissenschaftlichen Auseinandersetzung mit der Sprachkritik herrscht die Deutung vor, daß die sprachkritische Handhabung des Problems der Richtigkeit auf wissenschaftlich überholten Positionen des 19. Jahrhunderts beruht, in Sonderheit auf den sprachtheoretischen Grundlagen und methodischen Prinzipien der traditionellen Philologie und der historischen und vergleichenden Sprachwissenschaft, und durch eine unzulässige Vermischung synchronischer und diachronischer 
Sprachbetrachtung charakterisiert sei. Diese Deutung hat viel für sich. Zum einen findet man ja tatsāchlich solche historisierenden Argumente, zum anderen ist sie sehr gut mit der konservierenden, soziale Abgrenzung ermöglichenden Funktion der Sprachkritik vereinbar, von der im letzten Abschnitt die Rede war. Andererseits ist die Methode, die Richtigkeit des jeweils gegenwärtigen Sprachgebrauchs über den Rückgriff auf vergangene Sprachzustände zu bestimmen, gar nicht einmal vorherrschend, und sie wird von denen, bei denen man sie vor allem vermutet, den Sprachkritikern der letzten Jahrzehnte des 19. Jahrhunderts, z.B. Wustmann oder Engel, ausdrücklich kritisiert. Ich möchte deshalb die Aufmerksamkeit gezielt auf den zuletzt genannten Argumentationstyp, die Berufung auf die Struktur der Wirklichkeit bzw. das richtige Denken, lenken.

Neben der historischen Argumentation zieht sich durch das ganze 19. Jahrhundert eine andere, bei der Sprachkritiker Aussagen über die Richtigkeit aus einem bestimmten Verständnis der Beziehung zwischen Wort, Begriff und Ding, Sprache, Denken und Wirklichkeit gewinnen. Außerdem erweist sich die historische Argumentation nicht selten als Oberfläche, hinter der wieder das erwähnte Verständnis der Beziehung von Sprache - Denken - Wirklichkeit erkennbar wird. Wenn z.B. Richtigkeit der Sprache mit Goethe illustriert wird, dann oft deshalb, weil Goethe richtig und gut geschrieben habe, und das heißt im Rahmen des Argumentationsmusters, daß er seine Sprache in die richtige Relation zur Welt der Gedanken und zur Wirklichkeit gesetzt hat. Und dies stellt der Sprachkritiker nicht historisch, sondern auf einer einzelsprachunabhängigen und zeitlos gültigen Beurteilungsgrundlage fest. - Zunächst einige Beispiele, um die Art dieser Argumentation zu veranschaulichen.

(1) Ein gegenwärtiger Sprachglossenschreiber, Gerd Wittig (1980), nimmt sich einige Wörter vor, mit denen man im Deutschen, wie er sagt, ein fruchtbares Stück Land bezeichnen kann, nämlich Aue, Wiese, Flur, Anger und Weide. Daß die Ausdrücke sowohl stilistisch als auch historisch unterschiedlich markiert sind, kümmert ihn nicht. Er schickt sich an, für jeden Ausdruck eine eigene referentielle Bedeutung anzugeben. Um das im Fall von Anger und Weide bewerkstelligen zu können, bestimmt er Anger als "natürlichen Weideplatz mit schlechtem Boden". Wie kommt er darauf? Einen Hinweis gibt der Klammerzusatz "mit lateinisch ager $=$ Acker verwandt" - ein etymologisches Argument. Es hat jedoch im Kontext dieses Artikels eher die Funktion einer Ersatzlösung an einem Punkt, an dem es ihm nicht recht gelingt, das nachzuweisen, was er nachweisen möchte: daß jeder sprachliche Ausdruck in einer guten Sprache seine ihm eigene Referenz hat bzw. haben sollte. 
Damit befindet er sich ganz in Übereinstimmung mit seinem Kollegen vom "Tagesspiegel", der einige Wochen zuvor ausdrücklich geschrieben hatte, „daß eine gute Sprache keine überflüssigen Wörter kennt" (Tormen 1980). Was überfüssig ist, entscheidet sich im Vergleich mit der Wirklichkeit bzw. mit dem, was der jeweilige Autor der gedanklichen Unterscheidung für bedürftig hält. Faktisch erweist sich freilich in aller Regel, daß kein Wort überflüssig ist, denn der Zwang, eine direkte Korrelation zwischen Wörtern, Begriffen und Dingen herzustellen, ist so stark, $\mathrm{daB}$ zur Not ein Bedeutungsunterschied konstruiert wird; und dies kann, muß aber nicht geschehen durch Verweis auf die Etymologie.

(2) Der gleiche Autor beklagt in einer Glosse zu "Derselbe, der gleiche" (Wittig 1982) zunächst, daß der Ausdruck der gleiche, ,inzwischen überstark geworden sei". Das könnte Ausgangspunkt für eine historische Begründung der bekannten Bedeutungsunterscheidung sein. Wittig geht jedoch wieder einen anderen Weg. Er wendet sich der Wirklichkeit der Würste und Mützen zu und entdeckt dort Arten und Exemplare. Dann schwingt er sich auf die Ebene der Gedanken mit der Bemerkung, daß nes doch zumindest gedanklich sehr einfach [sei], dieselbe Wurst, die man soeben gekauft hat, wenn man sie in den Mund nehmen will, von der gleichen Wurst zu unterscheiden, die man schon gestern verzehrte". Die Schwierigkeit des dritten Schrittes, zu begründen nämlich, daß das gedanklich Unterschiedene auf der sprachlichen Ebene notwendig mit zwei verschiedenen Wörtern, und zwar genau mit den Ausdrücken derselbe und der gleiche unterschieden werden müsse, umgeht der Autor dadurch, daß der die beiden Ausdrücke schon in den Satz einschmuggelt, mit dem er die Notwendigkeit der gedanklichen Unterscheidung klärt, und damit voraussetzt, was begründet werden soll. Die Umgehung dieses heiklen Punktes ist übrigens typisch. Wenn ein anderer Glossist schreibt: „Ich kann mich nur zu jemandem hin bewegen [...], er aber nur zu mir her" (Ibel 1962, S. 104f.), dann verbleibt er sogar auf der ersten Ebene, und es klingt so, als würden einem die Knie versagen, wenn man es anders versuchte.

(3) Das dritte Beispiel, es stammt von Gerhard Storz, unterscheidet sich von den ersten beiden dadurch, daß es kein lexikalisches, sondern ein grammatisches Problem betrifft, und daß der Autor sich auf eine alte Tradition beruft.

Einen Konzessivsatz mit 'trotzdem' zu beginnen [...] ist falsch, weil die Verwendung der Partikel als Konjunktion gegen die Funktionalität der Syntax verstößt. Wir bedürfen des 'trotzdem' dort, wo wir etwas anderes, beispielsweise das 'obgleich', 'wiewohl' nicht brauchen können (Storz 1963, S. 185). 
Das Beispiel dient Storz dazu zu zeigen, daß Sprachrichtigkeit eine andere Grundlage besitze als die „bloße Gewöhnung”. Daher kann der Sprachgebrauch nicht Entscheidungsgrundlage sein. An seine Stelle tritt wiederum nicht die Geschichte, sondern die „Funktionalität der Syntax”, womit - das ist wichtig - nicht die Funktionalität speziell der Syntax des Deutschen gemeint ist, sondern menschlicher Sprache überhaupt. Er beruft sich auf die Grammatiker im Altertum und in der Neuzeit, die ,im Grunde das Funktionieren der Sprache" zu fassen suchten, „und deshalb lag ihrer Festsetzung der Sprachrichtigkeit das Bemühen um Sprachgerechtigkeit zu Grunde" (S. 285). Zu den allgemeinen Funktionsprinzipien gehört offenbar nicht nur wie in den ersten Beispielen eine Eins-zueins-Beziehung zwischen Ausdrücken und Inhalten, sondern auch, daß ein Ausdruck (trotzdem) nur eine grammatische Funktion erfüllt. Unterschiede in der Welt der Gedanken sollen generell ihre direkten Entsprechungen in der Welt der sprachlichen Ausdrücke haben.

(4) Wustmann spricht sich verschiedentlich gegen Versuche aus, das Problem der Sprachrichtigkeit geschichtlich zu lösen. Sein Sprachgefühl erlaubt es ihm sehr wohl, auch die Klassiker zu kritisieren, von denen er im Gegensatz zu vielen heutigen Nachfolgern weiß, daß sie nicht immer in seinem Sinne richtig und schön geschrieben haben. Anläßlich der Behandlung der Inversion nach und ironisiert er den ${ }_{n}$ wunderlichen Standpunkt", der es manchen Sprachgelehrten verbietet, die Inversion falsch zu finden, weil sie nämlich alt sei, und begegnet diesem Standpunkt mit dem Ausruf: "Als ob eine häßliche Spracherscheinung dadurch schöner würde, daB sie jahrhunderte alt ist" (Wustmann 1912, S. 307f.). Was hat er entgegenzusetzen? Ein Sprachgefühl, das auch bei ihm nicht selten Gefühl für Sprache überhaupt ist. Es ist z.B. am Werke, wenn er fast in allen Fällen richtig, in fast allen Fällen aber falsch findet. Die Präposition in müsse unmittelbar vor dem Wort stehen, das sie regiert: „Das ist so natürlich und selbstverständlich wie irgend etwas, es kann gar nicht anders sein"; "dieses Gesetz geht durch alle Sprachen, denn es ist in der Natur der Präpositionen begründet" (ebenda, S. 315).

(5) Als letztes Beispiel nehme ich noch einmal Schopenhauer, der die Verhunzung der Gegenwartssprache in zahllosen Einzelfallen im Vergleich mit Goethe oder den antiken Sprachen zu belegen sucht; der Urgrund seiner Sprachkritik ist m.E. aber auch eine Zeichentheorie, nach der "die Begriffe [...] Zeichen für Vorstellungen, wie Worte Zeichen für Begriffe" sind (Schopenhauer 1985, Bd. 1, S. 30), und eine bestimmte Auffassung über die optimale Realisierung dieser Beziehung. Dazu gehört u.a. wiederum die Eins-zu-eins-Beziehung zwischen sprachlicher Form und Bedeutung bzw. grammatischer Funktion. Auf dieser Grundlage erwei- 
sen sich bestimmte Wörter als unsinnig: „Das Wort Absolut ist an und für sich etwas ganz Unsinniges" (ebenda, S. 135). Warum? Es kann von keinem Objekt prädiziert werden. Umgekehrt werden Lücken im Wortschatz entdeckt, wenn für Begriffe Ausdrücke fehlen. Das ergibt u.a. ein Kriterium für die Aufnahme von Fremdwörtern: Fremdwörter sind zu begrüßen, wenn sie die Begriffe vermehren (Schopenhauer 1913, S. 633); die anderen sind nicht nur überflüssig, sondern schädlich, weil Synonymie der vollkommenen Sprache fremd ist. Gleiches gilt umgekehrt für die Polysemie: „Daß Ein Wort zwei verschiedene Bedeutungen hat, ist ein Uebelzustand, dem man stets entgegenarbeiten soll: sie [die Sprachverhunzer] befördern ihn!" (Schopenhauer 1985, Bd. 4, II, S. 40). Was für die Lexeme gilt, gilt auch für Derivationsmorpheme. Mit größter Strenge besteht er auf der je spezifischen Bedeutung von Vorlage und Vorlegung, Vergleich und Vergleichung, Entschluß, Entschließung, Entschlossenheit etc. (Bd. 4, II. S. 40, S. 47 u.o.), wie auch darauf, daB ein Ausdruck nicht Doppelfunktionen in verschiedenen Wortklassen erfüllen dürfe: Ausdrücke, die adjektivische Funktionen haben, dürfen nicht zugleich als Adverbien verwendet werden usw. (Bd. 4, II, S. 51f.).

Welche Rolle spielt nun die Geschichte in diesem Zusammenhang? Schopenhauer ist davon überzeugt, daB die ersten Menschen ,instinktgemä 8 ", wie die Biber ihren Bau und die Bienen ihre Waben, eine vollkommene Grammatik geschaffen haben, daf aber die Geschichte der Sprache seitdem ein Prozeb zunehmenden Verfalls ist (Schopenhauer 1851, S. 460468). Goethe kann deshalb Vorbild sein, weil er diesem Ursprung relativ näher steht als die Zeitgenossen; das Lateinische und Griechische ist dem Ursprung näher als Goethe, und das Sanskrit ist das Vollkommenste, was erreichbar ist, bevor die Entwicklung im Dunkel der Vorgeschichte verschwindet. Ich wage jedoch zu behaupten, daß es Schopenhauer in seinen sprachkritischen Maßstäben nicht erschüttert hätte, wenn ihn jemand davon überzeugt hätte, daß es die vollkommene Sprache, die seine Beurteilungsgrundlage ist, in Wirklichkeit nie gegeben hat.

Was die Beispiele deutlich machen sollten, ist ein Verfahren zur Bestimmung der Sprachrichtigkeit, das sich in folgender Weise charakterisieren läßt:

(1) Urteile über sprachliche Richtigkeit werden abgeleitet aus einer spezifischen Auffassung über die Beziehung zwischen Sprache, Denken und Wirklichkeit.

(2) In diesem Beziehungsgefüge ist Sprache nicht im Sinne von 'Einzelsprache', sondern von 'Sprache überhaupt' aufzufassen. Der Sprachkritiker, der sich auf sein Sprachgefühl beruft, beruft sich nicht auf ein Gefühl 
für seine Sprache, sondern auf universelle „Sprachgerechtigkeit” (Storz).

(3) $\mathrm{Da}$ dieses Gefühl eine einzelsprachunabhängige, letztlich sogar sprachunabhängige Grundlage hat, kann es sowohl auf den konkreten Sprachgebrauch in einer Sprache als auch auf das grammatische und lexikalische System der Einzelsprachen reagieren und letztere wertend vergleichen. Das erklärt vielleicht, warum Sprachkritiker zur Irritation der Linguisten zwischen Kritik an der Sprache und Kritik am Sprachgebrauch oft nicht unterscheiden.

(4) Untersucht man, worauf das Sprachgefühl ablehnend oder zustimmend reagiert, so scheint ein ganz zentraler, auf allen Ebenen der Sprache anwendbarer Maßstab die Kongruenz zwischen Wörtern, Gedanken und Dingen zu sein, die in der Auffassung der Sprachkritiker eine Einszu-eins-Beziehung zwischen Form und Inhalt bzw. Funktion erfordert. Dieses Kriterium ermöglicht es, die Frage nach der Sprachrichtigkeit, unabhängig vom Sprachgebrauch, sinnvoll zu stellen, ermöglicht in der Regel eine Ja-Nein-Entscheidung und erklärt, warum im Falle variierender Formen immer nur eine richtig sein kann.

(5) In den Beispielen selbst nicht deutlich geworden ist eine weitere in der Sprachkritik wirksame Vorstellung, die indes möglicherweise nicht notwendig mit den ersten vier Punkten verbunden ist. Sie läBt sich dahingehend formulieren, daß sprachliche Formen nicht nur eine je eigene, sondern eine ihnen wesentlich zugeordnete "wahre", "eigentliche" Bedeutung haben, der man durch Beachtung ihrer Wörtlichkeit oder durch Rückgriff auf die Historie habhaft werden kann. Das läuft tendenziell auf eine Deutung des Zusammenhangs von Form und Inhalt als nicht arbiträren hinaus.

Fragt man nach den sprachtheoretischen Wurzeln einer so argumentierenden Sprachkritik, so entfallen sowohl die strukturalistischen Sprachtheorien als auch die der historischen und vergleichenden Sprachwissenschaft des 19. Jahrhunderts. Nicht zu übersehen sind freilich die Affinitäten zu den Traditionen der philosophischen Sprachbetrachtung des 17. und 18. Jahrhunderts, die, wie schon früher erwähnt, in das 19. Jahrhundert weit hineinreichen. Ich weiß nicht, ob sich ein lückenloser Traditionszusammenhang vom 18. Jahrhundert bis heute nachweisen ließe, schließe die Möglichkeit aber nicht aus. Wie dem auch sei, an dem negativen sprachwissenschaftlichen Urteil über die sprachkritische Praxis des Umgangs mit dem Kriterium der Sprachrichtigkeit in den letzten 120-140 Jahren brauchte sich zunächst nichts zu ändern. In der Begründung für den Vorwurf, von wissenschaftlich unhaltbaren Vorstellungen über Charakter und Funktionieren von Sprache auszugehen, müßte der Verweis 
auf die Traditionen der historisch-vergleichenden Sprachwissenschaft nur um den Verweis auf die Traditionen der philosophischen Grammatik ergänzt werden.

\section{Sprachkritische, alltagsweltliche und linguistische Sprach- theorien: ein problematisches Verhältnis?}

Es scheint mir jedoch die Frage berechtigt, ob die skizzierten Vorstellungen über Sprache nicht eine Affinität zu verbreiteten alltagsweltlichen Sprachtheorien haben und, unabhängig von einem möglicherweise auch existierenden wissenschaftsgeschichtlichen Traditionszusammenhang, dort ihre Wurzeln haben und immer wieder neu erhalten. Das könnte auch der Sprachwissenschaft nicht gleichgültig sein, weil sie es dann nicht nur mit einigen unverbesserlichen Sprachschulmeistern zu tun hat, sondern mit den normalen Sprachteilhabern, deren Sprache sie zu beschreiben und deren Sprachbewußtsein sie zu rekonstruieren beansprucht.

Nun könnte man das nicht selten zu beobachtende Einverständnis zwischen Sprachkritikern und Sprachteilhabern, auch bezüglich der sprachtheoretischen Grundlagen, als Ergebnis eines über Generationen reichenden, vor allem über die Schule vermittelten Einflusses eben solcher Sprachkritiker deuten, im Verein mit Schulgrammatik und anderem abgesunkenen wissenschaftlichen Kulturgut. Die in Frage stehenden Auffassungen über Sprache wären, wenn sie Resultat dieses Einflusses sind, mit dem Begriff „alltagsweltlicher Sprachtheorien" falsch bezeichnet, wären sie doch Ergebnis einer fehlgeleiteten und angelernten Reflexion über Sprache, fern von und möglicherweise sogar im Gegensatz zu dem alltagsweltlich wirksamen, die kommunikative Praxis unreflektiert bestimmenden SprachbewuBtsein. Vorsicht ist geboten! Kein Zweifel, daß die Norm der Verwendung von brauchen ohne $z u$ in diesem Sinne angelernt ist und den, der sie hersagt, nicht davor bewahrt, im folgenden Satz brauchen ohne $z u$ zu gebrauchen, genauso wie es nicht unbedingt überrascht, daß jemand, der mir kürzlich versicherte, ihn störe die Hauptsatzkonstruktion nach weil, und er vermeide sie, in der Begründung für diese Selbsteinschätzung den Gegenstand seines Unbehagens reproduzierte.

Die in Frage stehenden Auffassungen über Sprache liegen möglicherweise jedoch tiefer. Nimmt man einerseits das linguistische Arbitraritätsprinzip und andererseits die nicht nur bei Sprachkritikern zu beobachtende Suche nach der "eigentlichen" Bedeutung eines Wortes, die zwar nicht die Faktizität einer nicht-arbiträren Beziehung von Ausdruck und Inhalt, aber die Möglichkeit einer solchen voraussetzt, und in der vor allem ein starkes Bedürfnis Ausdruck findet, es möge eine nicht-arbiträre Bezie- 
hung walten, so scheint mir die Frage, was angelernt und was wirkendes Sprachbewußtsein ist, nicht einfach zu entscheiden. Die Sprachgeschichte kennt ja sehr wohl die Prozesse der Sinngebung des Sinnlosen, genannt „Volksetymologie”, wie auch die Homonymenflucht und ähnliche Erscheinungen. Orientiert man sich an den Alltagstheorien über Sprache, kommt man auch in gewisse Schwierigkeiten mit neueren Ansätzen der linguistischen Semantik, in denen die Vorstellung, Wörter hätten eine kontextunabhängige Bedeutung, nur als unzulässige Hypostasierung überholter semantischer Theorien vorkommt. Auch diese Hypostasierung scheint mir dem alltagsweltlichen Sprachbewußtsein nicht fremd, und gegen dieses richtet das Urteil „wissenschaftlich überholt" nichts aus, kann man doch auch umgekehrt kritisch fragen, welche und wessen Sprache es denn ist, die Linguisten beschreiben, und von der gilt, daB es in ihr keine kontextunabhängigen Bedeutungen gibt, das Arbitraritätsprinzip waltet, Sprachrichtigkeit allein eine Sache des Gebrauchs ist usw.

Ich gebe also zu bedenken, ob nicht die Sprachkritiker z.T. etwas Ausdruck geben, was auch im alltagsweltlichen Sprachbewußtsein veraakert ist, und ob deshalb nicht die linguistische Kritik als Kritik an der Unwissenschaftlichkeit der Sprachkritik am Problem vorbeigeht, schließlich auch, ob man nicht - wie bei der Sprachkritik der ersten Hälfte des 19. Jahrhunderts - immer noch sagen könnte, auch die heutige sei, trotz ihrer Unzulänglichkeiten und der zuweilen kaum erträglichen Ignoranz, eine Form der Auseinandersetzung mit Sprache aus eigenem Recht.

\section{Literatur}

Abels, Kurt (1988): Zum Verhältnis von Deutschunterricht und Germanistik vom Anfang des 19. Jahthunderts bis um 1960. In: Oellers, Norbert (Hrsg.): Das Selbstverständnis der Germanistik. Aktuelle Diskussionen. (Vorträge des Germanistentages Berlin 1987, Bd. 1) Tübingen: Niemeyer, S. 105-119.

Bahner, Werner/Neumann, Werner (Hrsg.) (1985): Sprachwissenschaftliche Germanistik. Ihre Herausbildung und Begründung. Berlin: AkademieVerlag.

Blackall, Eric Albert (1966): Die Entwicklung des Deutschen zur Literatursprache 1700-1775. Stuttgart: Metzler.

Cherubim, Dieter (1983): Zur bürgerlichen Sprache des 19. Jahrhunderts. Historisch-pragmatische Skizze. In: Wirkendes Wort 30, S. 398-422.

Cherubim, Dieter/Mattheier, Klaus J. (1989): Vorwort. In: Cherubim, Dieter/Mattheier, Klaus J. (Hrsg.): Voraussetzungen und Grundlagen der 
Gegenwartssprache. Sprach- und sozialgeschichtliche Untersuchungen zum 19. Jahrhundert. Berlin/New York: W. de Gruyter, S. 1-9.

Dieckmann, Walther (Hrsg.) (1989): Reichthum und Armut deutscher Sprache. Reflexionen über den Zustand der deutschen Sprache im 19. Jahrhundert. Berlin/New York: W. de Gruyter.

Engel, Eduard (1922): Deutsche Stilkunst. 30. Aufl. Leipzig: G. Freytag (1. Aufl. 1912).

Glinz, Hans (1987): Die Sprachtheorien in und hinter den Lehrern und die Entwicklung der Sprachfähigkeit in den Schülern. In: Wimmer, Rainer (Hrsg.): Sprachtheorie. Der Sprachbegriff in Wissenschaft und Alltag. Jahrbuch 1986 des Instituts für deutsche Sprache. Düsseldorf. SchwannBagel, S. 206-236.

Hildebrand, Rudolf (1867): Vom deutschen Sprachunterricht in der Schule und von etlichem ganz Anderen, das doch damit zusammenhängt. In: Pädagogische Vorträge und Abhandlungen 1. Leipzig: J. Klinkhardt, S. 69-147.

Ibel, Rudolf (1962): Im Spiegel der Sprache. Kurzweilige und besinnliche Glossen zur deutschen Sprache. München: Heimeran.

Matthes, Günter (1989): Von Tag zu Tag: Alterserscheinungen. In: Der Tagesspiegel v. 25. Febr.

Naumann, Bernd (1986): Grammatik der deutschen Sprache zwischen 1781 und 1856. Die Kategorien der deutschen Grammatik in der Tradition von Johann Werner Meiner und Johann Christoph Adelung. Berlin: E. Schmidt.

Schopenhauer, Arthur (1851): Parerga und Paralipomena: kleine philosophische Schriften. Berlin: A.W. Hayn.

Schopenhauer, Arthur (1913): Parerga und Paralipomena. Kleine philosophische Schriften. Hrsg. v. Paul Dessau. (Sämtliche Werke, Bd. 5,2) München: Piper.

Schopenhauer, Arthur (1985): Der handschriftliche Nachlaß in fünf Bänden. Hrsg. v. Arthur Hübscher. München: dtv.

Schroeder, Otto (1906): Vom papiernen Stil. 6. Aufl. Leipzig/Berlin: B.G. Teubner (1. Aufl. 1889).

Sitta, Horst (1990): Defizit oder Entwicklung. Zum Sprachstand von Gymnasialabsolventen und Studenten. In: Stickel, Gerhard (Hrsg.): Deutsche 
Gegenwartssprache. Tendenzen und Perspektiven. Berlin/New York: W. de Gruyter, S. 233-254.

Storz, Gerhard (1963): Absichten und Grenzen der Sprachkritik. In: Figuren und Prospekte. Ausblicke auf Dichter und Mimen, Sprache und Landschaft. Stuttgart: Klett, S. 281-291.

Süskind, W.E. (1986): Gedanken zur Sprachpflege. In: Moser, Hugo (Hrsg.): Sprachnorm, Sprachpflege, Sprachkritik. Jahrbuch 1966/1967 (Sprache der Gegenwart 2) Düsseldorf: Schwann, S. 191-203.

Tormen, Ernst (1980): Sprachprivatissimum. In: Der Tagesspiegel v. 17. 8.

Wehler, Hans-Ulrich (1987): Deutsche Gesellschaftsgeschichte. Zweiter Band: Von der Reformära bis zur industriellen und politischen „Deutschen Doppelrevolution" 1815-1845/49. München: C.H. Beck.

Wissenschaftler widersprechen These vom Verfall der deutschen Sprache. „Leistungen von Schülern und Studenten sind gestiegen". In: Der Tagesspiegel v. 24. Febr. 1989.

Wittig, Gerhard (1980): Sprachprivatissimum. In: Der Tagesspiegel v. 26. 10.

Wittig, Gerhard (1982): Sprachprivatissimum: Derselbe, der gleiche. In: Der Tagesspiegel v. 31. 1 .

Wustmann, Gustav (1912): Allerhand Sprachdummheiten. Kleine deutsche Grammatik des Zweifelhaften, des Falschen und des HäBlichen. 6. Aufi. Straßburg: K.J. Trübner (1. Aufl. 1891). 